УДК 339.13

$10.17213 / 2075-2067-2021-3-116-125$

\title{
МАРКЕТИНГ ПРОСТРАНСТВЕННОГО ВЗАИМОДЕЙСТВИЯ В СОЦИАЛЬНО-ИНФРАСТРУКТУРНОМ РАЗВИТИИ ТЕРРИТОРИИ
}

\author{
(C) 2021 г. В. А. Бондаренко, Н. В. Гузенко, Т. С. Романишина \\ Ростовский государственный экономический университет (РИНХ), \\ 2. Ростов-на-Дону, Россия
}

\begin{abstract}
Целью исследования выступает выляление роли маркетинга пространственного взаимодействия (как конщепџии и ее практического воплощения) в осуществлении сочиально-инфраструктурного развития территории на базе создания маркетинг ориентированной инфраструктурыл.

Методологическая база исследования. В ралках исследования роли маркетинга пространственного взаимодействия в сочиально-инфраструктурном территориальном развитии мы опираемся на содержательный анализ профильных источников литературы, отражающих эволючионирование маркетинговой конщепџии, становление концепџии маркетинга пространственного взаимодействия, восприятия сочиумом изелевых установок конщепции устойчивого развития в рамках преобразования ожидаемых эффектов. Нами исследованы существующие точки зрения на формирование маркетинг ориентированной инфраструктуры и оправданности оперирования понятием социально-инфраструктурного развития территории. В статье использовались такие методы исследования, как метод литературного поиска, метод аналогий, метод содержсательного и компаративного анализа, метод синтеза полученных данных.

Результаты исследования. Важным результатом исследования является обоснование подхода на сочиально-инфраструктурное развитие территории в новых условиях, детерминируемых иелями устойчивого развития и маркетинговой парадигмой пространственного взаимодействия. Мы приходим к выводу, что инфраструктура в регионе является рыночно ориентированной, если она формируется, исходя из запросов существующих сейчас потребителей территориального продукта. В случае ее ориентачии на будущих потребителей (в широком смысле этого слова) она становится маркетинг ориентированной.

Перспективу исследования составляет маркетинговая ориентаџия, которая проявляется в контексте целевых установок устойчивого функционирования соииума и экономики, что задает определенные высокие требования социально-инфраструктурному развитию территорий.
\end{abstract}

Ключевые слова: маркетинг; пространственное взаимодействие; маркетинговая конщепция; сочиально-инфраструктурное развитие; территория.

\section{MARKETING OF SPATIAL INTERACTION IN THE SOCIAL AND INFRASTRUCTURAL DEVELOPMENT OF THE TERRITORY}

\author{
(C) 2021 V. A. Bondarenko, N. V. Guzenko, T. S. Romanishina
}

Rostov State University of Economics (RSUE), Rostov-on-Don, Russia

The purpose of the study is to identify the role of spatial interaction marketing (as a concept and its practical implementation) in the implementation of social and infrastructural development of the territory based on the creation of marketing-oriented infrastructure. 
The methodological base of the study. As part of the study of the role of spatial interaction marketing in socio-infrastructural territorial development, we rely on a meaningful analysis of specialized literature sources that reflect the evolution of the marketing concept, the formation of the concept of spatial interaction marketing, the perception of the society of the goals of the concept of sustainable development within the transformation of expected effects. We have investigated the existing points of view on the formation of a marketing-oriented infrastructure and the justification for operating with the concept of social and infrastructural development of the territory. The article uses such research methods as the method of literary search, the method of analogies, the method of meaningful and comparative analysis, the method of synthesis of the obtained data.

The results of the study. An important result of the research is the substantiation of the approach to the social and infrastructural development of the territory in the new conditions determined by the sustainable development goals and the marketing paradigm of spatial interaction. We conclude that the infrastructure in the region is market-oriented if it is formed based on the requests of the existing consumers of the territorial product. In the case of its orientation to future consumers (in the broadest sense of the word) it becomes marketing-oriented.

The prospect of the study is marketing orientation, which is manifested in the context of the targets of sustainable functioning of society and the economy, which sets certain high requirements for the social and infrastructural development of territories.

Key words: marketing; spatial interaction; marketing concept; social and infrastructural development; territory.

Введение. В рамках исследовательского контура мы определяем в объеме, достаточном для целевых ориентиров данной аналитической работы, сложившееся понимание концепции маркетинга пространственного взаимодействия в сопричастности с социально-инфраструктурным развитием территории [12]. Это предполагает одновременно с выявлением сути концепции маркетинга пространственного взаимодействия понимание эволюционирования маркетинговой теории в связи с изменениями условий хозяйствования, оценок успешности деловой активности (метрик), ориентированных на общественные интересы, наряду с интересами бизнеса и населения в соотнесении с установками социума на устойчивое развитие.

Мы обоснованно полагаем, что в современной ситуации, когда территориальное развитие подчинено ориентации на текущие потребности социума и бизнеса, интересы будущих поколений, ценности устойчивого эволюционирования социума, маркетинг в своем воплощении, не направленном на интенсификацию личного (неосознанного, избыточного) потребления, а в формате развертывания социально-этичной концепции, предполагающей взаимодействие заинтересованных сторон на долгосрочной основе и учет общественных интересов помимо желания извлекать прибыль, становится неотьемлемой составляющей планирования желательного состояния региональных социально-экономических систем. Эти обстоятельства актуализируют аналитическое изучение данного вопроса.

Материалы и методы. В рамках исследования роли маркетинга пространственного взаимодействия в социально-инфраструктурном территориальном развитии мы опираемся на содержательный анализ профильных источников литературы, отражающих эволюционирование маркетинговой концепции, становление концепции маркетинга пространственного взаимодействия, восприятия социумом целевых установок концепции устойчивого развития в рамках преобразования ожидаемых эффектов. Нами исследованы существующие точки зрения на формирование маркетинг ориентированной инфраструктуры и оправданности оперирования понятием социально-инфраструктурного развития территории. В статье использовались такие ме- 
тоды исследования, как метод литературного поиска, метод аналогий, метод содержательного и компаративного анализа, метод синтеза полученных данных.

Обсуждение. Согласно сформировавшейся позиции, официальным документам и проводимым управленческим решениям Россия является социально ориентированным государством, реализующим социальную политику, нацеленную на повышение качества жизни населения. В данном контексте целесообразно говорить о социальноинфраструктурном развитии территорий, которое подразумевает под собой выбор такой траектории положительного эволюционирования, в рамках которой зримо проявляется нацеленность на формирование маркетинг ориентированной инфраструктуры, создаваемой, обновляемой и эксплуатируемой в рамках изначальной ориентации на потребности (текущие и прогнозируемые) заинтересованных лиц территории [12].

Ранее нами обосновывалась концепция рыночно ориентированной и маркетинг ориентированной инфраструктуры [4], которая уже в фазе своего проектирования, введения и непосредственной эксплуатации нацелена на максимально возможное удовлетворение потребностей целевых аудиторий террито- рии, бизнеса, власти, населения, привлекаемых извне граждан и инвесторов, деловых партнеров территории. Социальная направленность в формате социально-инфраструктурного развития территории проявляется в инфраструктурном (материальном, институциональном, персональном) обеспечении, способствующем привлечению/удержанию населения, связывающего свой карьерный рост и будущую личную и профессиональную жизнь с определенной территорией в качестве осознанного выбора, оцениваемого в качестве лучшего из возможных вариантов.

Отметим также, что маркетинговая составляющая обеспечения территориального развития, при котором анализируется качество, результативность взаимодействия субъектов в определенном пространстве, нашла отражение, например, в работах Г. Багиева, А. Шульги, А. Пинчука, развивающих идейное наполнение концепции маркетинга пространственного взаимодействия $[1,2,3,10$, 11]. Мы нацелены на развитие данных научных взглядов и сопряжение этого процесса с пониманием необходимости проектирования маркетинг ориентированной инфраструктуры в рамках социально-инфраструктурного развития территории.

Целью статьи выступает выявление роли маркетинга пространственного взаимо-

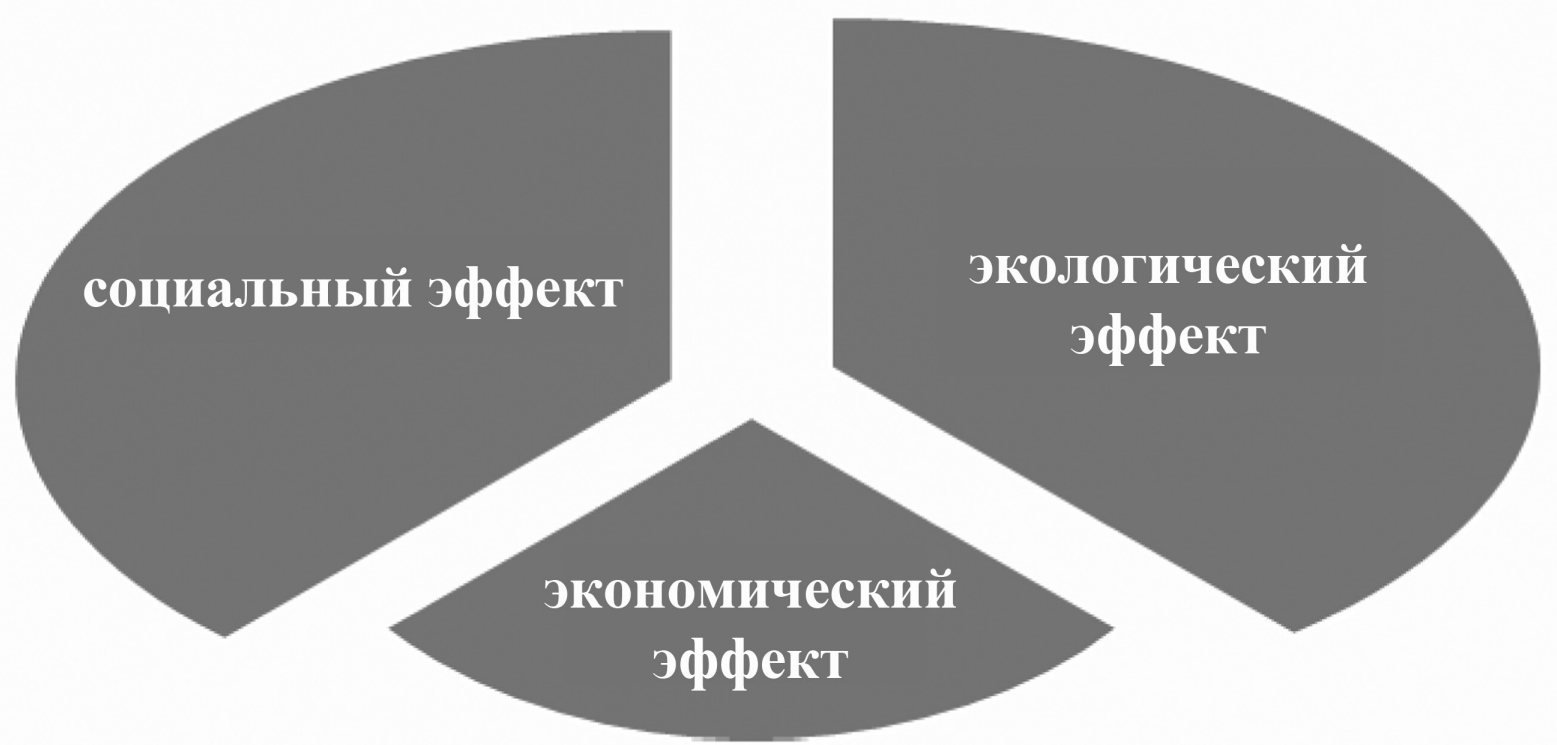

Рис. 1. Приверженность к достижению трех искомых эффектов в функционировании социально-экономических систем в рамках ценностных ориентиров устойчивого развития $[5,9]$ 
действия (как концепции и ее практического воплощения) в осуществлении социальноинфраструктурного развития территории на базе создания маркетинг ориентированной инфраструктуры.

Результаты. Маркетинг взаимодействия заинтересованных в развитии территории сторон (включающий в себя социально-этичный компонент за счет приверженности целевым установкам устойчивого развития), положенный в основу положительного эволюционирования территории, определяет возможности создания маркетинг ориентированной инфраструктуры в регионе и детерминирует общий вектор социально-инфраструктурных преобразований.

В основу ценностных ориентиров устойчивого развития положена одновременная приверженность к достижению трех искомых эффектов (рисунок 1).

Уточним, что в территориальном маркетинге целевыми аудиториями, заинтересованными в развитии территории, выступают органы власти в регионе, бизнес, локализованный в регионе, предприятия и организа-

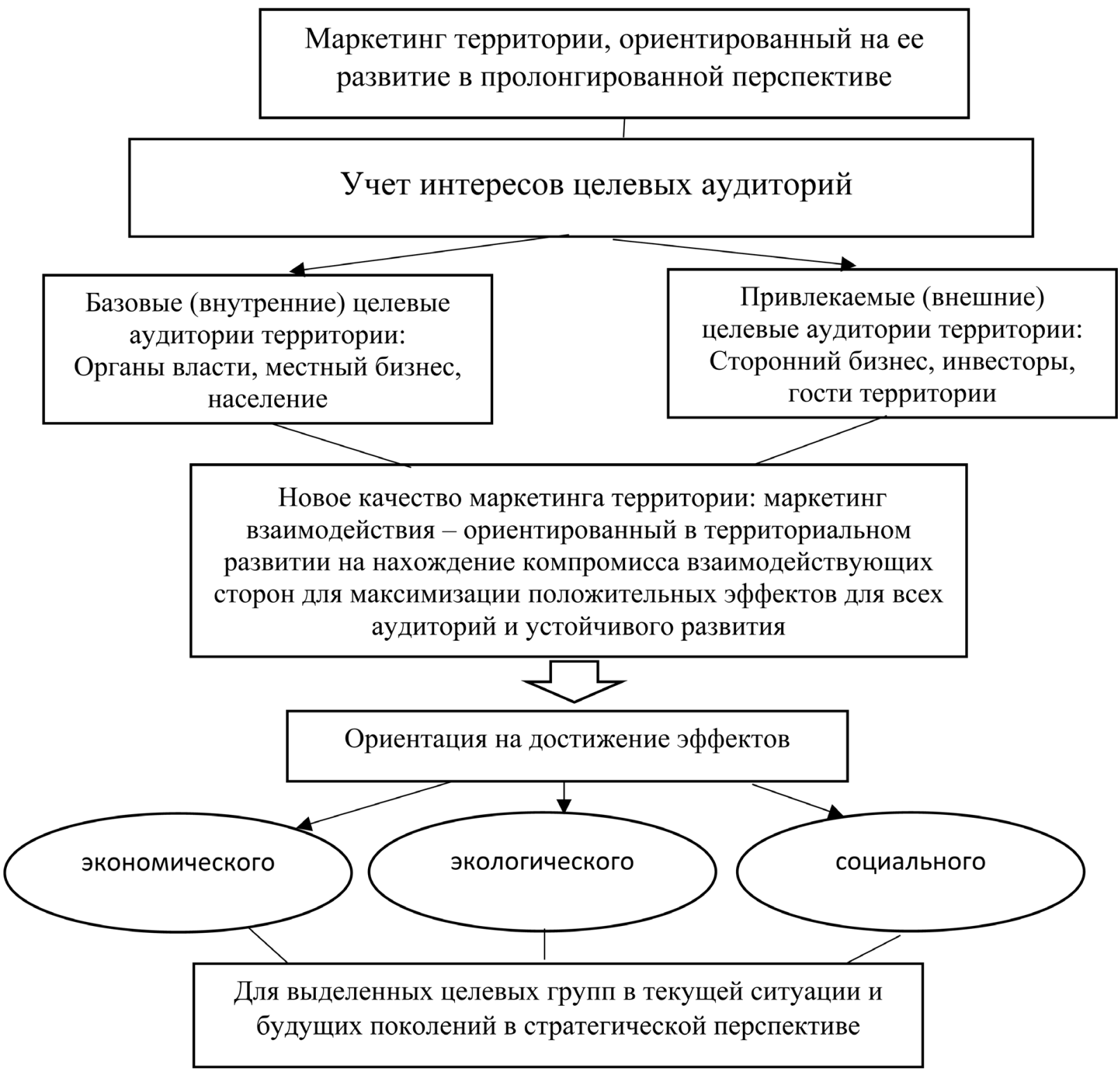

Рис. 2. Ориентация на маркетинг взаимодействия в рамках нацеленности на устойчивое развитие и принимаемых решений по эволюционированию территории (составлено авторами) 
ции, местное сообщество (население, связывающее свою карьерную и личную судьбу с регионом), а также внешние инвесторы, сторонний бизнес, партнеры территории и индивидуальные потребители территориального продукта, например, гости региона.

Соответственно, речь в данном исследовании идет о вовлечении маркетинговой теории в практику территориального развития, а именно построении маркетингового взаимодействия между заинтересованными лицами определенной территории, их долгосрочные взаимовыгодные контакты, априорно ориентированные не только на экономические эффекты и частные интересы игроков, но социальные и экологические эффекты для территории и вмещающего ее сообщества (рисунок 2).

Отметим также, что в рамках детерминируемых 16 целей устойчивого развития в данном исследовании наиболее целесообразно выделить социальные проекты, такие как доступное здравоохранение и образование, инновации и инфраструктура, устойчивые города и населенные пункты [13].

В данном контексте становится очевидным, что сама устойчивая модель, которая детерминирована социумом в качестве желательной, актуализирует проблематику социально-инфраструктурного развития территорий. Она сочетается с рыночной моделью региональной экономики, которая в соприкосновении с целевыми установками устойчивого развития переориентируется на рациональное осознанное потребление, положительные эффекты для социума, не только в экономике, но и экологии и социальных аспектах.

Это обстоятельство трансформирует теорию и практику маркетинга в территориальном развитии, которая далее при воплощении в реальность сама трансформирует

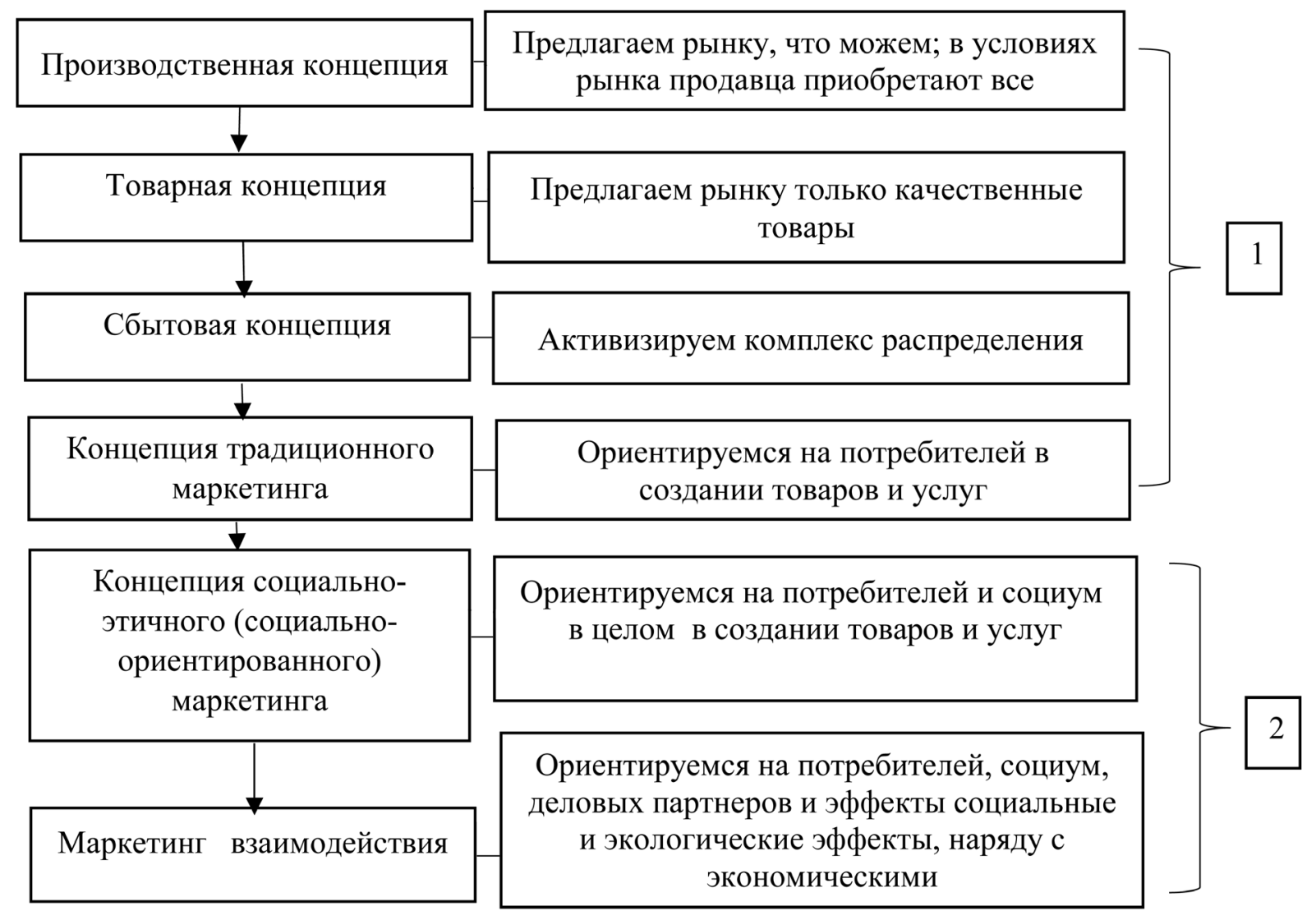

Рис. 3. Визуализация эволюционирования маркетинговой концепции [3]:

1 - концепции, ориентированные на подхлестывание спроса для интенсификации прибыли; 2 - концепции, ориентированные на обдуманное потребление

(на текущих и будущих потребителей) 
социально-экономический ландшафт. Такие трансформации становятся возможными и обоснованными, поскольку меняется потребитель, который выступает основой преломления маркетинговой теории и осуществления маркетинговой практики. В сложившейся ситуации речь идет о текущих потребителях (целевых аудиториях территории) и одновременно будущих потребителях, пролонгированные интересы которых учитываются априорно без их реального проявления в настоящий момент. Именно это обстоятельство делает потребление осознанным, сохраняющим возможность реализовывать потребительскую функцию в дальнейшем новым поколениям индивидов. Такая заинтересованность в создании возможностей потребления в будущем и проявляется в оценке социальных и экологических рисков и нацеленности на их купирование в результате собственных обдуманных актов потребления, граничащих с рациональным самоограничением (что проявляется в различных отраслях и сферах деятельности, исследуемых отечественными и зарубежными учеными $[6,7,8,14])$.

Нацеленность на удовлетворение потребностей текущих и будущих аудиторий в эволюционировании территорий приводит к практической актуализации вопросов социально-инфраструктурного развития, когда создаваемая маркетинг ориентированная инфраструктура позволяет выбирать вектор будущего функционирования для социальноэкономической системы региона.

Соответственно, если проанализировать эволюцию маркетинговой концепции от возникновения до современного наполнения и понимания, то данные проявления станут понятны (рисунок 3 ).

Отметим, что исследователи склонны саму парадигму взаимодействия рассматривать в качестве обновленного (компромиссного) подхода к созданию ценности в условиях изменившихся ориентиров социальноэкономического развития (рисунок 4).

Получается, что в случае дискретных отношений цели взаимодействующих субъектов разнонаправлены, ориентированы на достижение частных интересов без учета запросов социума. В случае маркетинга долгосрочного взаимодействия стороны (партнеры, нашем случае - заинтересованные лица территории) сотрудничают с акцентом на компромиссные цели, сочетающие экономические, социальные и экологические эффекты в формате социально-инфраструктурного развития.

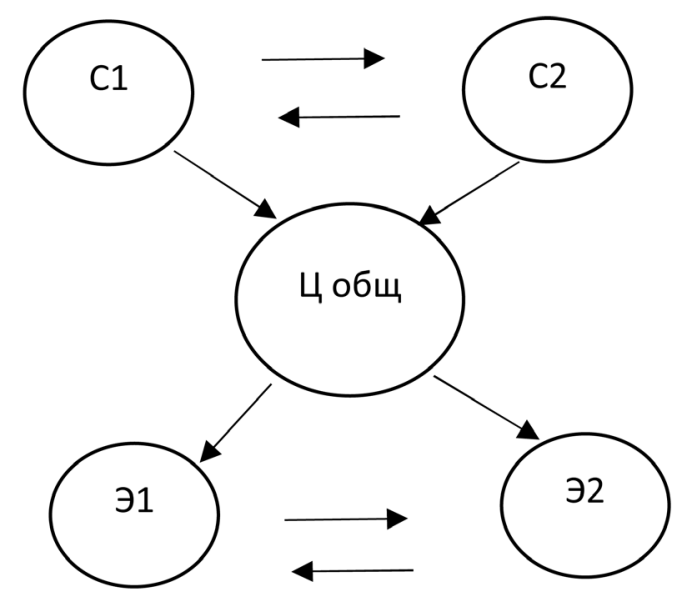

б

Рис. 4. Визуализация дифференциации дискретных отношений и долгосрочного взаимодействия субъектов

в условиях изменившихся ориентиров социально-экономического развития [2]:

a - отношение (дискретное), персонифицированное;

б - взаимодействие (длительное), предполагающее общие ценностные установки и обязательства 
Заключение. Применительно к территориальному развитию принято выделять концепцию маркетинга пространственного взаимодействия, которая трактуется специалистами как «социально-экономические отношения между различными субъектами региональной экономики в нацеленности на нахождение, производство, распределение и потребление ценностей» в рамках территориального объединения. Эти ценности создаются на компромиссной основе с учетом интересов будущих поколений, будущих потребителей территории, по данной причине в создании инфраструктуры для различных нужд эволюционирования региональной экономики учитываются текущие и прогнозные потребности.

В этом проявляется подход на социально-инфраструктурное развитие территории в новых условиях, детерминируемых целями устойчивого развития и маркетинговой парадигмой пространственного взаимодействия. Поясним свою мысль. Инфраструктура в регионе является рыночно ориентированной, если она формируется, исходя из запросов существующих сейчас потребителей территориального продукта. В случае ее ориентации на будущих потребителей (в широком смысле этого слова) она становится маркетинг ориентированной. Маркетинговая ориентация проявляется в контексте целевых установок устойчивого функционирования социума и экономики, что задает определенные высокие требования социально-инфраструктурному развитию территорий.

\section{Литература}

1. Багиев Г.Л., Пинчук А.В., Серова Е.Г., Шульга А.О. К вопросу формирования концепции маркетинга пространственного взаимодействия // ПСЭ. — 2012. — №9. C. 219-224.

2. Багиев Г.Л., Шульга А.О. Концепция маркетинга пространственного взаимодействия: измерение и оценка эффективности // ПСЭ. - 2010. — №12. - С. 262-265.

3. Багиев Г.Л., Асаул А.Н. Организация предпринимательской деятельности. Учебное пособие / Под общей ред. проф. Г. Л. Багиева. - СПб.: Изд-во СПбГУЭФ, 2001. $231 \mathrm{c}$.
4. Бондаренко В.А. Маркетинговое сопровождение инфраструктуры внешнеэкономической деятельности: эволюция, современное состояние и трансформационные тенденции. Монография. - Ростов-н/Д, 2010. - $236 \mathrm{c}$.

5. Бондаренко В.А., Ефременко И.Н., Гузенко Н. В. Вопросы теоретического обоснования зеленой экономики и ее перспективы для России // Финансовые исследования. 2019. - №2 (63). - С. 23-31.

6. Бондаренко В.А., Гузенко Н. В., Ларионов B.A. Вопросы применения гостиницами зеленых инноваций в рамках проявившихся потребительских предпочтений // Вестник Ростовского государственного экономического университета (РИНХ). - 2020. №1 (69). - С. 17-23.

7. Бондаренко В.А., Ларионов В.А. Исследование ценностных установок российских потребителей услуг «зеленых» гостиниц// Практический маркетинг. — 2021. №1 (287). - C. 3-11.

8. Костоглодов Д.Д., Бондаренко В.А., Гуськов А.И. Экологический маркетинг в развитии рынка органических продуктов питания в России: эволюция, современное состояние, перспективные тенденции (региональный аспект). Монография. - Ростов-наДону, 2014. - 136 с.

9. Лясковская Е. А., Григорьева К. А. Формирование зеленой экономики и устойчивость развития страны и регионов // Вестник ЮУрГУ. Серия «Экономика и менеджмент». - 2018. - Т. 12. - №1. - С. 15-22.

10. Пинчук А.В. Обоснование и оценка экономической эффективности перспективной концепции маркетинга // Известия СПбГЭУ. 2014. 一 №2 (86). - С. 112-115.

11. Пинчук A.B. Бифуркация концепции маркетинга и инструментарий обеспечения структурной стабильности маркетинговых систем // ПСЭ. — №9. - С. 230-234.

12. Романишина Т.С., Дадаян Н.А., Полуянова Н.В. Проблемы неравномерности регионального развития в обосновании маркетинговой ориентации социально-инфраструктурного обеспечения территории // Экономика устойчивого развития. - 2021. №2 (46).

13. Цели в области устойчивого развития [Электронный ресурс]. - Режим доступа: 
https://www.un.org/sustainabledevelopment/ru/ sustainable-development-goals/ (Дата обращения: 20.02.2021).

14. Meskhi B., Bondarenko V., Efremenko I., Larionov V., Rudoy D., Olshevskaya A. Technical, technological and managerial solutions in ensuring environmental safety / IOP Conference Series: Materials Science and Engineering. The collection of conference materials. Voronezh State University of Engineering Technologies. - 2020. - P. 12100 .

\section{References}

1. Bagiev G.L., Pinchuk A.V., Serova E. G., Shul'ga A. O. K voprosu formirovanija koncepcii marketinga prostranstvennogo vzaimodejstvija [To the question of formation of the concept of marketing in spatial interaction] // PSJe. 2012. — №9. — Pp. 219-224.

2. Bagiev G.L., Shul'ga A.O. Koncepcija marketinga prostranstvennogo vzaimodejstvija: izmerenie i ocenka jeffektivnosti [The marketing concept of spatial interaction: the measurement and evaluation of effectiveness] // PSJe. 2010. — №12. — Pp. 262-265.

3. Bagiev G.L., Asaul A.N. Organizacija predprinimatel'skoj dejatel'nosti. Uchebnoe posobie [Organization of Business activity. Traning manual] / In prof. G. L. Bagiev (eds.). - Saint Petersburg: Izd-vo SPbGUJeF, 2001. — 231 p.

4. Bondarenko V.A. Marketingovoe soprovozhdenie infrastruktury vneshnejekonomicheskoj dejatel'nosti: jevoljucija, sovremennoe sostojanie i transformacionnye tendencii. Monografija [Marketing support of the infrastructure of foreign economic activity: evolution, current state and transformational trends. Monograph]. - Rostov-on-Don, 2010. - $236 \mathrm{p}$.

5. Bondarenko V.A., Efremenko I.N., Guzenko N.V. Voprosy teoreticheskogo obosnovanija zelenoj jekonomiki i ee perspektivy dlja Rossii [Questions of theoretical justification of the green economy and its prospects for Russia] // Finansovye issledovanija [Financial research]. - 2019. - №2 (63). - Pp. 23-31.

6. Bondarenko V.A., Guzenko N.V., Larionov V.A. Voprosy primenenija gostinicami zelenyh innovacij $\mathrm{v}$ ramkah projavivshihsja potrebitel'skih predpochtenij [Questions of the use of green innovations by hotels within the framework of manifested consumer preferenc- es] // Vestnik Rostovskogo gosudarstvennogo jekonomicheskogo universiteta (RINH) [Vestnik of the Rostov State University of Economics (RSUE)]. — 2020. — №1 (69). - Pp. 17-23.

7. Bondarenko V.A., Larionov V.A. Issledovanie cennostnyh ustanovok rossijskih potrebitelej uslug «zelenyh» gostinic [Research of value settings of Russian consumers of services of «green» hotels] // Prakticheskij marketing [Practical marketing]. — 2021. — №1 (287). — Pp. 3-11.

8. Kostoglodov D.D., Bondarenko V.A., Gus'kov A.I. Jekologicheskij marketing v razvitii rynka organicheskih produktov pitanija v Rossii: jevoljucija, sovremennoe sostojanie, perspektivnye tendencii (regional'nyj aspekt). Monografija [Ecological marketing in the development of the organic food market in Russia: evolution, current state, promising trends (regional aspect). Monograph]. — Rostov-on-Don, 2014. - $136 \mathrm{p}$.

9. Ljaskovskaja E.A., Grigor'eva K.A. Formirovanie zelenoj jekonomiki i ustojchivost' razvitija strany i regionov [Formation of the green economy and sustainability of the development of the country and regions] // Vestnik JuUrGU. Serija «Jekonomika i menedzhment»[Bulletin of the South Ural State University. Series «Economics and Management»]. — 2018. Vol. 12. - №1. - Pp. 15-22.

10. Pinchuk A. V. Obosnovanie i ocenka jekonomicheskoj jeffektivnosti perspektivnoj koncepcii marketinga [Rationale and evaluation of economic efficiency perspective of the concept of marketing] // Izvestija SPbGJeU [Bulletin of St. Petersburg State Economic University]. 2014. — №2 (86). — Pp. 112-115.

11. Pinchuk A. V. Bifurkacija koncepcii marketinga i instrumentarij obespechenija strukturnoj stabil'nosti marketingovyh sistem [Bifurcation of marketing concepts and tools to ensure structural stability of the marketing system] // PSJe. - №9. - Pp. 230-234.

12. Romanishina T.S., Dadajan N.A., Polujanova $N$. $V$. Problemy neravnomernosti regional'nogo razvitija $\mathrm{v}$ obosnovanii marketingovoj orientacii social'no-infrastrukturnogo obespechenija territorii [Problems of uneven regional development in the justification of marketing orientation, socio-infrastructural support site] // Jekonomika ustojchivogo razvitija [The economy of sustainable development]. — 2021. №2 (46). 
13. Celi v oblasti ustojchivogo razvitija [Sustainable Development Goals] [Jelektronnyj resurs]. — URL: https://www.un.org/sustainabledevelopment/ru/sustainable-developmentgoals/ (Date accessed: 20.02.2021).

14. Meskhi B., Bondarenko V., Efremenko I., Larionov V., Rudoy D., Olshevskaya A. Techni- cal, technological and managerial solutions in ensuring environmental safety / IOP Conference Series: Materials Science and Engineering. The collection of conference materials. Voronezh State University of Engineering Technologies. - 2020. - P. 12100.

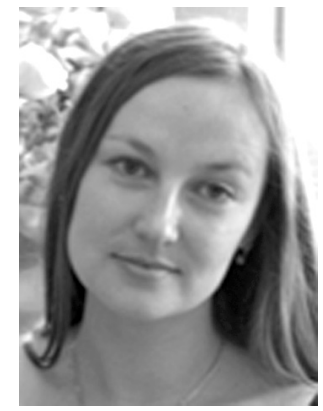

Бондаренко Виктория Андреевна - доктор экономических наук, доцент, заведующая кафедрой «Маркетинг и реклама» Ростовского государственного экономического университета (РИНХ).

Bondarenko Victoria Andreevna - Doctor of Economic Sciences, Associate Professor, Head of the Department «Marketing and Advertising», Rostov State University of Economics (RSUE).

344002, г. Ростов-на-Дону, ул. Б. Садовая, 69

69 B. Sadovaya st., 344002, Rostov-on-Don, Russia

E-mail: b14v@yandex.ru

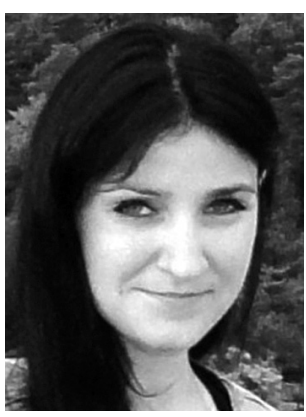

Гузенко Наталья Владимировна - кандидат экономических наук, доцент кафедры «Коммерция и логистика» Ростовского государственного экономического университета (РИНХ).

Guzenko Natalia Vladimirovna - Candidate of Economic Sciences, Associate Professor of the Department «Commerce and Logistics», Rostov State University of Economics (RSUE).

344002, г. Ростов-на-Дону, ул. Б. Садовая, 69 69 B. Sadovaya st., 344002, Rostov-on-Don, Russia E-mail:musamav@mail.ru 


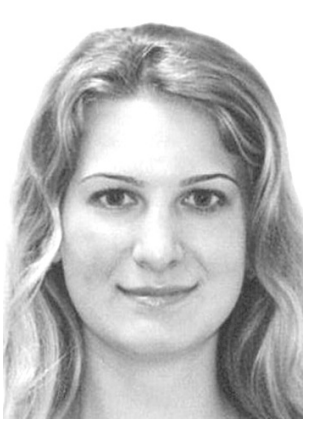

Романишина Татьяна Сергеевна - кандидат экономических наук, доцент института сервисных технологий Российского государственного университета туризма и сервиса.

Romanishina Tatiana Sergeevna - Candidate of Economic Sciences, Associate Professor of Institute of Service Technologies, Russian State University of Tourism and Service.

344002, г. Ростов-на-Дону, ул. Б. Садовая, 69

69 B. Sadovaya st., 344002, Rostov-on-Don, Russia

E-mail: tankarom@yandex.ru 